\title{
IMPACTO DE LA MULTIMODALIDAD EN LA COMPRENSIÓN LECTORA DE TEXTOS NARRATIVOS EN INGLÉS COMO LENGUA EXTRANJERA (L2) EN ESTUDIANTES UNIVERSITARIOS
}

\author{
Fernando Vera Millalén \\ Universidad de Aconcagua (Chile)
}

\begin{abstract}
RESUMEN: En el siguiente trabajo se busca comparar el efecto de las pruebas monomodales frente a las multimodales en el índice de comprensión lectora de textos narrativos en L2 que alcanzan los estudiantes de una universidad chilena privada. Para este efecto, se realizó un cuasi-experimento, con una prueba de comprensión lectora en formato monomodal y otra en formato multimodal, y grupos intactos. Al grupo experimental se les administró una prueba en formato multimodal, mientras que al grupo control se le administró una prueba en formato monomodal. La experiencia avala la necesidad de integrar textos multimodales en la comprensión lectora en L2. Los resultados corroboran esta hipótesis, pues, los estudiantes alcanzan niveles de comprensión más altos, en pruebas de comprensión lectora, con formato multimodal.
\end{abstract}

PALABRAS CLAVE: Aprendizaje de lenguas extranjeras, multimodalidad, textos narrativos, comprensión lectora.

\section{IMPACT OF MULTIMODALITY IN READING COMPREHENSION OF NARRATIVE TEXTS IN ENGLISH AS A FOREIGN LANGUAGE (EFL) IN UNDERGRADUATE STUDENTS}

\begin{abstract}
This research seeks to compare the effect that monomodal and multimodal tests have on the reading comprehension level of English-written narrative texts that EFL learners achieve at a private Chilean university. For this purpose, a quasi-experiment was performed, using a monomodal reading comprehension test and another multimodal one, with intact groups. The experimental group took the multimodal format test, while the control group took the monomodal format test. The interest of this research focused on the need to integrate multimodal texts in L2 reading comprehension. The results endorse the hypothesis that students reach higher comprehension levels in multimodal reading comprehension tests.
\end{abstract}


KEYWORDS: Foreign language learning, multimodality, narrative texts, reading comprehension.

Recibido: 26/05/2014

Aceptado: 11/11/2014

\section{INTRODUCCIÓN}

Sin lugar a dudas, el aprendizaje de una lengua extranjera, especialmente del inglés, constituye una de las competencias que todo profesional requiere hoy en día, como herramienta de competitividad. La enseñanza y aprendizaje de una lengua extranjera (L2) es uno de los grandes desafíos curriculares de las Instituciones de Educación Superior (IES) chilenas, que buscan añadir valor al currículo formativo de pregrado para así dar una respuesta más efectiva a la necesidades socioeducativas surgidas de los procesos de globalización, las comunicaciones internacionales, la transfronterización de la educación, y el desarrollo de las Tecnologías de la Información y Comunicación (TIC).

Dentro de este contexto, diversas universidades chilenas han incorporado en su currículo la enseñanza de inglés mediante el Enfoque Comunicativo y la utilización de diversos recursos tecnológicos, especialmente multimedia interactiva. Con esta metodología, se busca desarrollar la competencia comunicativa de los estudiantes y así contribuir a incrementar su movilidad y posterior empleabilidad. Comparativamente, desde la perspectiva tradicional de enseñanza, el aprendizaje de L2 ha estado sometido esencialmente a un enfoque centrado en el docente, que incluye el pizarrón, la grabadora o video, y fundamentalmente, el texto de estudio, con notables consecuencias de apatía, frustración y falta de interés entre los aprendices; actitudes que necesitan revertirse porque los estudiantes actuales son mucho más multimodales que los de antaño. Conviene destacar que el enfoque comunicativo persigue el desarrollo integral de las competencias comunicativas, tanto a nivel receptivo (comprensión auditiva y escrita) como productivo (expresión oral y escrita), con énfasis en el desarrollo de la producción oral.

Respondiendo a tales desafíos, la universidad en estudio implementa un Programa de Inglés Transversal, como proyecto innovador en todas las carreras de pregrado de sus sedes Machalí y Rancagua, en la Región del Libertador Bernardo O'Higgins. Es importante señalar que el currículo de inglés, de 6 niveles, de este proyecto responde a diversos estándares internacionales para el aprendizaje de L2, cuya columna vertebral, en su fase de autoaprendizaje, es la plataforma de multimedia interactiva English Discoveries, un producto de multimedia interactiva, que integra textos orales y escritos en formato multimodal.

Sin embargo, en las actividades evaluativas de comprensión lectora, tanto de textos en inglés como lengua extranjera (English as a Foreign Language: EFL) como en inglés para fines específicos (English for Special Purposes: ESP), se ha observado que los estudiantes presentan bajos niveles de rendimiento en pruebas construidas por los profesores en formato monomodal. Por tanto, resulta de alto interés investigativo averiguar las causas que estarían produciendo tales diferencias de comprensión lectora de textos narrativos en L2. 
Más específicamente, este estudio tiene un interés concreto en investigar sobre el aprendizaje multimodal y su impacto en la comprensión lectora de textos narrativos en L2 y tiene como objetivo general comparar el efecto de las pruebas monomodales frente a las multimodales en el índice de comprensión lectora de textos narrativos en L2, que alcanzan los estudiantes de una universidad chilena privada, mediante una prueba de comprensión lectora. En consecuencia, se espera que los resultados de este estudio sean una importante contribución al campo de la enseñanza de inglés en nuestro país, especialmente en lo relativo a la coherencia constructiva que es necesario que haya entre las formas de enseñar y las formas de evaluar.

\section{COMPRENSIÓN LECTORA EN L2}

Las investigaciones en el campo de la comprensión lectora en L2 plantean que hay dos factores que explican el desarrollo lector. Por un lado, está el factor específico de la lengua extranjera, tal como el conocimiento de vocabulario o de la gramática y, por el otro, está el factor de conocimiento general y transferible, como la conciencia metacognitiva relacionada con las estrategias lectoras, considerada un componente del conocimiento general de la lectura, que podría transferirse desde la lectura en la lengua materna (L1) a la lectura en L2. De este modo, algunos investigadores, como Schoonen, Hulstin y Bossers (1998) sostienen que los buenos lectores en L1 son también buenos lectores en L2. Por tanto, la conciencia metacognitiva en la comprensión lectora en L2 es vista como un factor transferible desde L1, lo que supone realizar más investigación empírica para examinar cómo operan los aspectos morfosintácticos, el vocabulario, la conciencia metacognitiva de las estrategias lectoras, y las estrategias metodológicas en el proceso de desarrollo de la lectura en L2. En particular, se hace necesario probar si las estrategias lectoras compensan la debilidad en la sintaxis y el vocabulario en L2.

Así, contrariamente a lo que ocurre en el desarrollo de la comprensión lectora en L1, en L2 los procesos son muy distintos. Al respecto, Koda (1994) plantea tres condiciones críticas que diferencian el proceso de lectura en L2 respecto de su contraparte en L1, que incluyen la experiencia lectora previa; la interferencia interlingüística (L1 versus L2); y el conocimiento lingüístico limitado que el lector tiene de la lengua meta. Estos factores podrían obstaculizar la comprensión lectora en L2.

Es más, cuando se lee en L2, el lector está en una posición psicológica muy diferente de la que ocupa en L1 (Ausubel, Novak y Hanesian, 2005). Esto quiere decir que el lector en L1 ya domina el vocabulario básico y el código sintáctico de su idioma y es capaz, por lo tanto, de leer, comprender y aplicar proposiciones sintácticas de manera aceptable.

Aparentemente, no ocurre lo mismo al leer un texto en L2, en donde los procesos de comprensión lectora presentan otros ritmos de procesamiento de la información textual. De hecho, la mayor parte de los especialistas concibe la lectura en L2 como un proceso interactivo. De este modo, según Bernhardt (1983, 1991), el lector interactúa con el texto para crear significado a medida que los procesos mentales operan en diferentes niveles. Esto supone que si en L1 se tiene un buen rendimiento y buena práctica lectora, en L2 se conseguiría una mejor comprensión textual. 
Por su parte, Barnett (1989) en sus investigaciones sobre las estrategias lectoras en L2 encontró que los lectores efectivos tienden a leer un pasaje completo y luego vuelven a leerlo con el propósito de pensar sobre lo que conocen sobre el tema, hipotetizan sobre lo que podría venir luego, y adivinan el significado de las palabras desconocidas, concluyendo que los lectores menos efectivos se centran en el significado de palabras individuales, ponen mayor atención a la estructura del texto, vuelven a leer solamente pasajes difíciles, nunca o rara vez realizan hipótesis, y se resisten a saltar $\mathrm{u}$ omitir las palabras desconocidas.

\section{ESTRATEGIAS LECTORAS EN L2}

Las investigaciones en torno a las estrategias lectoras en L2 demuestran que los aprendices de lenguas extranjeras utilizan diversos caminos para abordar o interactuar con el texto escrito (Barnett, 1989). Así, por ejemplo, los lectores novatos o menos exitosos intentan procesar el texto palabra a palabra, mientras que los más diestros evitan el procesamiento a nivel de palabras y optan por centrarse en el significado y las relaciones lógicas, incluso descartando, en cierto modo, el texto impreso. Al respecto, Widdowson (1978) plantea que resulta interesante tener en cuenta las distintas estrategias lectoras que ocurren en un contexto comunicativo, con un propósito comunicativo, tales como:

- Lectura extensiva: lectura por placer, con énfasis en la comprensión general.

- Lectura intensiva: lectura cuidadosa que permite encontrar detalles específicos del texto. Muy utilizada en el tratamiento lexical del texto.

- Lectura rápida o skimming: lectura rápida que permite darse cuenta de qué trata el texto.

- Lectura exploradora o scanning: lectura que permite localizar información específica en un texto. Generalmente se usa en horarios, itinerarios, gráficos, etc.

Debido a lo anterior, generalmente el desarrollo de la lectura guiada en L2 se aborda desde las así llamadas estrategias lectoras. En este sentido, es común que los profesores de lenguas extranjeras comiencen con actividades de pre-lectura (pre-reading) antes de proceder con la lectura misma. Entre éstas se podrían citar discusiones previas sobre el tema, presentación de fotografías, revisión de vocabulario clave, organización textual (palabras, oraciones principales, frases, títulos y subtítulos), etc.

En este contexto, el dominio lexical es esencial para alcanzar un buen nivel de comprensión textual en L2. Según Laufer (1986), para lograr una comprensión mínima se requiere conocer un $95 \%$ o más de las palabras que se encuentran en un texto. Por su parte, Hosenfeld (1979) señala que el nivel de comprensión textual depende de cuán bien las variables del lector (interés en el texto, propósito para leer dicho texto, conocimiento del tema, habilidad para aprender una lengua extranjera, toma de conciencia sobre el proceso lector, y disposición a asumir riesgos) interactúan con las variables del texto (tipo de texto, estructura, sintaxis, y vocabulario).

Ahora bien, para el aprendizaje de una lengua extranjera, Carrell y Carson (1997) recomiendan siempre la lectura extensiva como una estrategia favorecedora de la comprensión lectora. Cabe señalar que este tipo de lectura se refiere a leer grandes 
cantidades de material o lecturas más largas, como, por ejemplo, libros enteros, con énfasis en el significado de lo que se lee más que en el lenguaje. La idea es incentivar la lectura fuera de la sala de clases. Como se puede apreciar, la lectura extensiva como estrategia de aprendizaje de L2, se basa esencialmente en la habilidad lectora que se tiene en L1. En esta misma línea argumentativa, Susser y Robb (1990) sostienen que la lectura extensiva es efectiva, si se replican los modelos que se utilizan cuando se aprende a leer en la lengua materna. En esta línea, una estrategia simple para mejorar la comprensión lectora es la propuesta por Palincsar y Brown (1984) en su programa de mejora de la habilidad lectora. Estos autores señalan cuatro estrategias que podrían extrapolarse muy bien en L2, tales como, resumir, clarificar dificultades, formular una pregunta y hacer predicciones sobre el contenido. Aunque, esta estrategia se podría aplicar tal cual al aprendizaje de L2, se propone un algoritmo en donde el lector identifica significados (palabras nuevas y desconocidas), resume, formula preguntas por párrafo y hace predicciones, tanto de contenido como de significados.

Por otra parte, y dado que la comprensión lectora en L2 requiere de un esfuerzo extra por parte del lector, Chun y Pass (1996) proponen facilitar este proceso incorporando al texto sonidos, ayudas visuales y animaciones, los que, en su conjunto, juegan un importante rol en la adquisición de vocabulario y en la comprensión textual global. Es lo que actualmente se conoce como multimodalidad, que aborda el aprendizaje en L2 desde diversos modos combinados. En esta misma línea, Jewitt y Kress (2003) ponen su atención en el componente multimodal, pues el texto plano se regula por la lógica de la secuencia temporal, mientras que el texto multimodal se rige por la espacialidad, composición y simultaneidad.

Del mismo modo, Serafini $(2011,2012)$ sostiene que la incorporación de elementos multimodales expande la comprensión lectora en L2, debido a que los jóvenes, hoy en día, están cada vez más expuestos a textos que contienen imágenes visuales que los ayudan a construir significado y a adquirir estrategias lectoras diferentes a las tradicionales actividades cognitivas, tales como resumir, formular preguntas y predecir significado. En este sentido, la presente investigación pretende dar cuenta de los procesos emergentes de comprensión lectora en L2, que integra los complementos visuales de la información textual.

\section{Aprendizaje MULTIMODAL}

Es un hecho que los textos vienen hoy en día en diferentes formatos y que hacen uso de diversos modos de comunicación, por lo que la comprensión lectora en L2 ya no puede ser pensada únicamente desde la perspectiva de los textos planos o monomodales. De este modo, la multimodalidad puede definirse de manera simple como la diversidad de formas de hacer o concebir una acción comunicativa. Adicionalmente, podemos afirmar que, en el contexto del aula, se observan diversos modos de comunicación que están activos, ya sea de manera oral o escrita, durante el desarrollo de las diversas actividades de enseñanza y aprendizaje. Más aún, desde el enfoque comunicativo de lenguas, la multimodalidad supone la utilización de diversos recursos didácticos y la estimulación de los propios estudiantes como un 
importante recurso lingüístico. Esta concepción multimodal del aula tiene grandes repercusiones para el aprendizaje (Kress y Van Leeuwen, 2001).

En consecuencia, el aprendizaje ya no depende central o predominantemente del lenguaje (Kress y Van Leeuwen, 2001). Este planteamiento supone que el aprendizaje ocurre cuando el aprendiz se involucra muy dinámicamente en una actividad que incorpora diversos modos de presentación y comunicación, y en la cual el discurso oral y el discurso escrito son sólo uno de los diversos modos involucrados en una determinada actividad. Es más, el uso de los recursos computacionales para la exploración, lectura y construcción de significados obliga a considerar la variable multimodal (Matthewman, Blight y Davies, 2004). Como se ha demostrado, esta variable tiene gran incidencia en la interpretación, deducción o construcción de significados (Kress, 2003).

Por otra parte, las interacciones entre los individuos no sólo pueden describirse desde el lenguaje verbal sino que también desde los modos visuales y accionales de la comunicación (Kress y Van Leeuwen, 2001). A lo que podríamos agregar que hoy en día ya no estamos limitados a un modo único (escrito) sino que podemos ofrecer a los estudiantes una combinación de modos, incorporando así el potencial de significados implicados en el discurso, la escritura y la presentación visual (Hampel, 2002). Desde la perspectiva pedagógica, este hecho nos obliga a establecer una importante distinción entre los modos comunicacionales cotidianos y los modos comunicacionales inducidos. Llamaremos modos comunicacionales cotidianos a aquellos que son parte de nuestra vida, y, por lo tanto, de los cuales no somos totalmente conscientes. A la inversa, los modos comunicacionales inducidos se refieren a los recursos multimodales que se disponen como parte de una determinada metodología orientada a facilitar el aprendizaje de una materia específica. En otras palabras, estos modos inducidos generan, en este caso, una atención consciente al componente multimodal. En relación con lo que hemos denominado modos comunicacionales inducidos, podemos afirmar que los recursos multimodales contribuyen a crear nuevos estilos de pensamiento y aprendizaje (Kress y Van Leeuwen, 2001).

Lo anterior tiene importantes repercusiones para la enseñanza y aprendizaje de idiomas, y en especial del inglés, el cual, en términos comparativos, exhibe uno de los mayores desarrollos en el campo de la lingüística aplicada. Por esta razón, es común encontrar textos de estudios con abundante incrustación de ilustraciones y sistemas de software con componentes multimediales, es decir, sonido e imágenes. Más aún, los efectos semióticos en los procesos cognitivos son muy relevantes a la hora de diseñar una apuesta curricular en L2 basada en recursos multimodales.

En tal sentido, hay que precisar que, desde la perspectiva comunicativa, las funciones y efectos de las ilustraciones en un texto, pueden derivarse de características, tales como, las imágenes que ilustran algunos elementos del texto y las imágenes que no sólo representan diversos elementos extra textuales, sino que además aportan información adicional no lingüística. Al respecto, Mayer y Sims (1994) sostienen que presentar el texto con material visual puede ayudar al lector de L2 a construir inferencias entre la presentación verbal y la presentación visual, y así aprender más efectivamente. 
En este sentido, podemos afirmar que cuando se lee un texto ilustrado se generan efectos motivacionales importantes en el lector de L2, por lo que los textos ilustrados facilitarían el proceso lector, aumentando así el placer por su lectura (Willows y Houghton, 1987). Por consiguiente, cuando hablamos de recursos pedagógicos en el área de lenguas extranjeras, resulta más atractivo y motivador abordar un texto narrativo multimodal que una versión plana o monomodal.

\section{TeXtos MULTIMODAles}

Hoy en día, los ambientes de aprendizaje de los estudiantes se ven colmados de textos en formatos visuales, electrónicos y digitales, los que reciben el nombre de textos multimodales (Kress, 2003). Muchas de las investigaciones en el campo de la enseñanza y aprendizaje de lenguas extranjeras apuntan a generar teorías en relación con las nuevas pedagogías que se requieren para responder a un ambiente de aprendizaje que ha cambiado, pues actualmente los textos incluyen elementos multimodales.

En general, se llama texto multimodal a aquel que contiene componentes textuales (lingüísticos) y componentes visuales (no lingüísticos). Adicionalmente, este tipo de texto podría contener componentes multimediales, tales como, imágenes, animación y sonido. Cualquiera sea el caso, los textos multimodales han co-existido desde hace mucho tiempo. La diferencia entre ayer y hoy es que antes primaba la lógica de la escritura por sobre la lógica de la imagen. Sin embargo, en plena era de los medios, hoy predomina la lógica de la imagen por sobre la lógica de la escritura. Cabe señalar que, como sostiene Albers (2007), los modos de comunicación visual, oral, escrita, gesticular, musical, y así sucesivamente, facilitan la interpretación y presentación de significado. Esto quiere decir que en cualquier texto multimodal los diversos modos operan de manera conjunta para representar un significado global.

Desde el punto de vista de los modos de procesamiento textual, lo anterior supone que la lectura de un texto monomodal es más inductiva mientras que la lectura de un texto multimodal es más deductiva. Esto significa que cuando un lector inicia el procesamiento de un texto multimodal, en cierto modo se prepara para un entorno particular, que aparte de los elementos lingüísticos le ofrecerá elementos visuales y semióticos, con lo cual el procesamiento de la información resultará más amigable.

En sus estudios sobre los textos multimodales, Kress (2003) ha encontrado interesantes hallazgos. Por ejemplo, podría haber diferencias en la disposición a la lectura de textos multimodales relacionados con la edad, el género y otras. Al respecto, quienes han sido socializados en un mundo de medios contemporáneos podrían ver la pantalla como su punto de referencia para el desarrollo de las estrategias de lectura, afirmando que, en plena era digital, cuando el texto se despliega en la pantalla éste queda subordinado a la lógica de la pantalla e imagen (Kress, 2003). Al respecto, hay dos perspectivas teóricas que se potencian mutuamente con el propósito de definir el marco conceptual de la lectura de textos multimodales, conocidas como presentación textual en formato multimodal no impreso y presentación textual en formato multimodal impreso. La primera perspectiva aprovecha las recientes investigaciones y conceptualizaciones con respecto a la incorporación de imágenes y textos multimodales, principalmente a través de multimedia interactiva. Cabe señalar que los textos multimodales son aquellos que emplean más de un modo de comunicar significados a tra- 
vés de una sincronización de múltiples modos. Es decir, pueden incorporar lenguaje hablado y escrito, e imágenes estáticas y en movimiento. Por tanto, pueden producirse en papel o en una pantalla electrónica e incorporar sonido.

Es importante señalar que muchos de los textos multimodales que comúnmente se emplean en contextos educativos se presentan en forma impresa a través de textos ilustrados, diarios y revistas (Gee, 2007; Jewtitt y Kress, 2003). Por su parte, los textos multimodales en formato no impreso incluyen películas, video $y$, de manera creciente, aquellos textos que se presentan a través de pantallas electrónicas, tales como multimedia, Internet, y medios digitales como CD-ROM, o DVD.

Dada la importancia que han cobrado los recursos informáticos, resulta evidente que la multimodalidad es una impulsora de la adquisición de una lengua extranjera, pues, como sostiene Kress (2003), la pantalla constituye hoy el medio de comunicación de la actual generación. Lo anterior supone grandes desafíos para los profesores de idiomas en sus tareas de selección o elaboración de material visual y auditivo, y la correspondiente evaluación de los aprendizajes esperados en L2. En otras palabras, la perspectiva multimodal requiere de profesores con competencias en el diseño y desarrollo curricular, que consideren en sus decisiones de micro implementación, los intereses y experiencias previas de sus estudiantes (Albers, 2007). Sin embargo, el desafío también plantea la necesidad de incluir una enseñanza multimodal efectiva que aborde las posibilidades que ofrecen los textos multimodales para el aprendizaje de L2.

Este nuevo escenario supone preparar a profesores de inglés que sean capaces de desarrollar, de manera integral, las diversas destrezas lingüísticas de recepción (leer y escuchar) y de producción (hablar y escribir), pues los elementos multimodales potencian no sólo la adquisición de estructuras morfosintácticas y léxico asociado en L2, sino que también su aplicación práctica en diversas tareas comunicativas. Así, todo parece indicar que existe una necesidad de integrar elementos extras textuales, como imagen y sonido en los textos narrativos en L2, como una forma de facilitar la comprensión lectora que alcanzan los aprendices de L2. De hecho, los textos de estudio EFL son una clase de material didáctico, eminentemente comunicativo, que incluyen una gran variedad de imágenes para así ayudar a activar los conocimientos previos del aprendiz, con lo cual se estimula la asociación que éste hace entre lo visual y lo escrito.

Por otra parte, los textos de estudio EFL son una necesidad para la mayoría de los profesores de inglés, debido a que estos presentan la lengua para su uso efectivo en una variedad de contextos comunicativos, ya que, más que presentar lecciones gramaticales, ofrecen oportunidades reales para abordar temas interesantes orientados a gatillar la comunicación y el trabajo colaborativo entre los estudiantes.

Tal enfoque comunicativo es el mismo que se utiliza en los textos multimediales para el aprendizaje de lenguajes extranjeras, en los cuales se agregan imágenes, animaciones y sonido, con lo cual se facilita aún más la asociación que hacen los aprendices entre significados y elementos multimodales, pues, como bien señalan Rodríguez, Ochoa y Parker (2006), desde la perspectiva tecnológica, la multimedia permite estructurar mejor la información, mientras que, desde la perspectiva cogni- 
tiva, ésta facilita que el estudiante se convierta en un sujeto activo, involucrado en la construcción de conocimientos, a partir de los datos que procesa.

En términos de la comprensión lectora en L2, se podría decir que el procesamiento de textos multimodales en formato impreso difiere cualitativamente del procesamiento de textos multimodales en formato multimedial. Por ejemplo, un estudiante que lee un libro ilustrado necesitará procesar simultáneamente el mensaje que aparece tanto en las palabras, las ilustraciones, como en los gráficos. Sin embargo, esta situación mejoraría con la incorporación de la pantalla electrónica o digital que agrega combinaciones de movimiento y sonido, como elementos extra textuales que facilitarían la comprensión, tanto lectora como auditiva en L2.

A través de diversos estudios, Kress y Van Leeuwen (2001) han desafiado las nociones de los enfoques tradicionales de enseñanza, cuyo énfasis se centra en el formato impreso monomodal, con una propuesta curricular que incorpora textos multimodales en formatos digitales, que posibilitan una mayor interacción del lector con el texto en L2. Estos investigadores sostienen que la enseñanza tradicional de L2 no es suficiente para satisfacer las prácticas metodológicas que se necesitan en plena era de la información. A partir de estos aportes al campo de la lingüística aplicada, se ha alertado sobre las nuevas formas de comunicación que la pantalla y los textos multimodales en formato digital están generando en las nuevas generaciones de estudiantes, lo que supone elaborar currículos de L2 que integran esta variable multimodal.

Hoy en día, los aprendices del nuevo milenio han crecido junto al computador y las nuevas formas digitales de diagramación y presentación textual disponibles para el aprendizaje de L2. Esto ha hecho que el mensaje visual haya cobrado mayor importancia cuando se analiza la comprensión del texto escrito. De ahí que actualmente los expertos en desarrollo curricular de L2 planteen la necesidad de integrar el aprendizaje multimodal y los sistemas de aprendizaje basado en ordenador (CALL: Computer-Assisted Language Learning), en el aprendizaje de L2, pues la comprensión textual no puede ser entendida sin la noción de la competencia discursiva del lector de la imagen $u$ otros elementos extra textuales.

En síntesis, parece ser que los nuevos tipos de textos en L2 requieren de otras conceptualizaciones y otras formas de diseñar los materiales didácticos, ya que, como afirma Kress (2003), hay diferencias significativas entre las palabras aisladas y las rodeadas de imágenes. En términos simples, al desarrollar la comprensión lectora en L2 es preciso considerar que la lógica del discurso (uso correcto de reglas gramaticales) demanda mucho más tiempo y secuencia de comprensión que la lógica de la imagen, que involucra espacio y simultaneidad. De este modo, la lectura de textos visuales implica un proceso distinto a la lectura de palabras planas. Este mismo autor sostiene que las escuelas estimulan la lógica de la escritura, mientras que las experiencias de vida de los niños y jóvenes contemporáneos se fundamentan en la lógica de la imagen y la lógica de la pantalla. Como desafío metodológico, lo anterior supondría incluir aspectos del código visual en los textos narrativos en L2, ya que los elementos extra textuales portan información procesable, que contribuye cualitativamente al procesamiento de la información textual de cada lector. 


\section{Metodología}

El presente estudio se enfoca desde una perspectiva cuantitativa y posee un alcance correlacional. El diseño es un cuasi experimento con post test y grupo control, en donde la información se obtiene mediante la aplicación de dos pruebas de comprensión lectora de textos narrativos en L2, una de ellas en formato monomodal y la otra en formato multimodal, a un grupo de estudiantes universitarios. De acuerdo con el diseño, en esta investigación se aplicó la prueba en formato monomodal al grupo control y la prueba en formato multimodal al grupo experimental. De esta manera, el estímulo experimental se encuentra ya incluido en las pruebas aplicadas, pues se comparan los resultados en función del formato de presentación textual y se analiza la incorporación de elementos extra textuales (formato multimodal) y sus efectos en la comprensión lectora en L2.

De esta forma, la hipótesis a contrastar en el estudio será: La aplicación de pruebas de comprensión lectora en L2, con formato multimodal, se asocia a mayores puntuaciones y mayor tasa de aprobados que la aplicación de pruebas en formato monomodal.

\section{Participantes}

La muestra total consta de 53 participantes (38 mujeres y 15 hombres), de los cuales 15 corresponden a la carrera de Psicología (PSI), 12 a la carrera de Trabajo Social (TSO), 6 a la carrera de Pedagogía en Educación Parvularia (PEP) y 20 a la carrera de Pedagogía en Educación Básica (PEB), pertenecientes al nivel A1 (ALTE 1) del Programa de Inglés de una universidad chilena privada. De esta muestra, 33 participantes corresponden al grupo experimental y 20 al grupo control, como se refleja en la tabla 1.

Tabla 1. Distribución de la muestra

\begin{tabular}{|l|c|c|c|c|}
\hline \multirow{2}{*}{ Secciones originales } & Edad & \multicolumn{2}{|c|}{ Género } & \multirow{2}{*}{ Total } \\
\cline { 2 - 4 } & Promedio & Masculino & Femenino & \\
\hline Grupo experimental & & & & \\
PSI & 25 & 08 & 07 & 15 \\
TSO-PEP & 24 & 02 & 16 & 18 \\
\hline Grupo control (PEB) & 23 & 03 & 17 & 20 \\
\hline
\end{tabular}

\section{Instrumentos}

Para la recogida de datos se utilizaron dos instrumentos, con el mismo contenido a evaluar, pero en formatos de presentación distintos, es decir, una prueba de comprensión lectora en formato multimodal, aplicada al grupo experimental y otra prueba en formato monomodal, aplicada al grupo control. La prueba corresponde a un instrumento de muestra de exámenes Cambridge, nivel PET (Primary English Test), que tiene como objetivo evaluar la comprensión lectora de los candidatos. De este modo, se eligieron los textos "Dough Allan, Wildlife Cameraman" y "The 
Ancient Islands of Orkley", ambos adaptados a un formato multimodal y los mismos en formato monomodal, es decir, sin elementos extra textuales. Los referidos textos corresponden al subtest Entry Level Certificate in English (ESOL) Entry 3, 100/2031/7. PET Handbook, UCLES 2004. Sample Paper.

La sección de la prueba estandarizada elegida evalúa la comprensión lectora de los candidatos a partir de los siguientes ítems:

- Ítem de selección múltiple correspondiente a cinco preguntas, cada una de ellas con cuatro afirmaciones, de las cuales el candidato debe seleccionar la que mejor responda a la pregunta-guía. Este ítem contiene cinco preguntas-guía.

- Ítem de selección múltiple correspondiente a 10 afirmaciones que se realizan sobre el texto, de las cuales el candidato debe marcar con $A$ las afirmaciones correctas y con $B$ las afirmaciones incorrectas.

Por lo tanto, las pruebas, en su conjunto, contienen 15 preguntas de comprensión lectora sobre textos narrativos correspondientes al nivel PET de los candidatos.

Dado que los instrumentos utilizados para la recogida de datos, son parte del conjunto de subpruebas de muestra del Examen Internacional Cambridge, correspondiente al nivel PET, se logró finalmente construir instrumentos confiables y pertinentes para los propósitos de la presente investigación. Para entregar mayor validez a los instrumentos se sometieron a un análisis de juicio de expertos, para de esta forma entregarle validez de contenido a los mismos. Los profesionales que analizaron los instrumentos corresponden a un grupo de especialistas en el área TEFL.

Cabe señalar que la puntuación máxima de la prueba, en ambos formatos, es de 75 puntos, con una exigencia de $60 \%$ para alcanzar la condición de aprobado, equivalentes a una puntuación de 45 puntos, que se aplica a cada grupo de estudiantes, en una misma ocasión.

\section{Resultados}

Se hará un tratamiento estadístico de los datos mediante el programa STATA (Statistics/Data Analysis), versión 10.0. El análisis estará dividido en dos partes, uno descriptivo y otro inferencial. En el análisis descriptivo se expondrán los estadísticos descriptivos (media, desviación estándar) calculados a partir de las puntuaciones obtenidas por los sujetos separados por grupos; además se analizará la frecuencia absoluta y porcentual por grupo de los aprobados y reprobados, tomando como patrón de rendimiento mínimo aceptable (PREMA) el 60\% indicado previamente. Para el análisis inferencial se utilizará una prueba paramétrica de comparación de medias (T de Student para muestras independientes), vale decir, se contrastará la puntuación media que obtenga el grupo experimental respecto del grupo control para evaluar si la diferencia es significativamente distinta. Además, se realizará una prueba de proporciones para dos muestras, para así decidir si la proporción de aprobados y suspensos entre ambos grupos es significativamente distinta. Ambas pruebas se realizarán con un intervalo de confianza de $95 \%$ y un nivel de significancia de $5 \%$. Mediante estas pruebas estadísticas se buscará contrastar la hipótesis del estudio que sostiene que la aplicación de pruebas de comprensión lectora en L2, 
con formato multimodal se asocia a mayores puntuaciones y mayor tasa de aprobados que la aplicación de pruebas en formato monomodal.

\section{Análisis descriptivo}

La puntuación promedio del grupo experimental fue 55,6/75 puntos, con una desviación estándar de 10,059. Referente a lo acontecido con el grupo control se tiene que la puntuación promedio fue de 38,8/75 puntos, con una desviación estándar de 7,926 (tabla 2). Respecto de los niveles de aprobados y suspensos por grupo, se aprecia que de los 33 aprendices tomados del grupo experimental, 31 alcanzan la condición de aprobado (correspondiente al 93,9\% del total del grupo), mientras que de los 20 informantes del grupo control, 13 de ellos logran la condición de aprobado (correspondientes al 65\% del total del grupo), como se muestra en la tabla 3.

Tabla 2. Estadísticos descriptivos por grupo

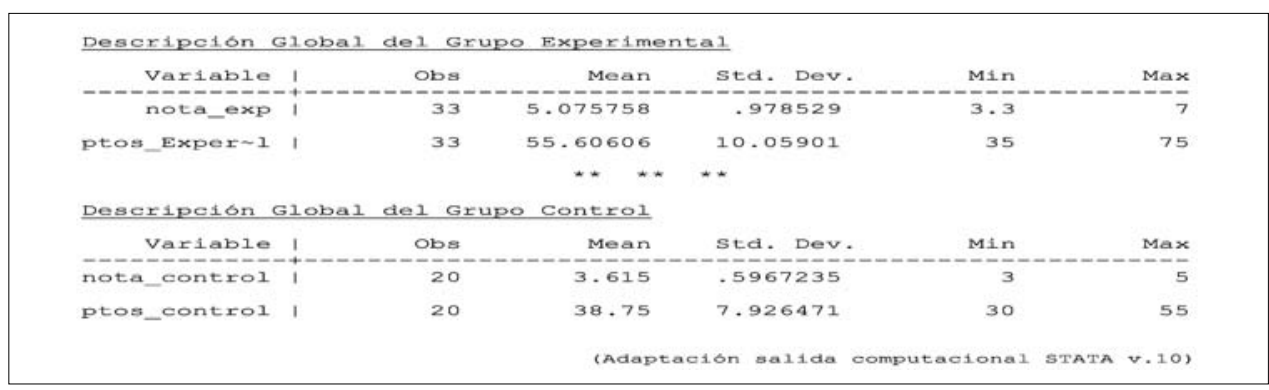

Tabla 3. Contingencia de aprobados/suspensos por grupo

\begin{tabular}{|c|c|c|c|}
\hline Condición & G-Experimental & C-Control & Total \\
\hline Aprobados & $31(93,9 \%)$ & $13(65 \%)$ & $44(83 \%)$ \\
\hline Suspensos & $2(6,1 \%)$ & $7(35 \%)$ & $9(17 \%)$ \\
\hline & $33(100 \%)$ & $20(100 \%)$ & $53(100 \%)$ \\
\hline
\end{tabular}

\section{Análisis inferencial}

Como se indicó previamente, para contrastar la hipótesis de estudio se realizará una prueba T de Student que comparará las medias de las puntuaciones obtenidas en ambos grupos en las pruebas, para así poder decidir si existe una diferencia significativa en dichas medias. La puntuación promedio obtenido en el grupo experimental fue de 55,6, mientras que en el grupo control fue de 38,8. Descriptivamente, se evidencia una diferencia a favor del grupo experimental, la cual es ratificada por el análisis inferencial, ya que la prueba $T$ de Student muestra un valor de $t=6,381$, con una significancia $p=0,000$ (tabla 4). Dado lo anterior se puede afirmar que existe una diferencia significativa entre las puntuaciones obtenidas por los dos grupos, es decir, que la puntuación obtenida por el grupo experimental es significativamente mayor que el obtenido por el grupo control. 
IMPACTO DE LA MULTIMODALIDAD EN LA COMPRENSIÓN LECTORA DE TEXTOS NARRATIVOS EN INGLÉS...

Tabla 4. T de Student para dos muestras independientes

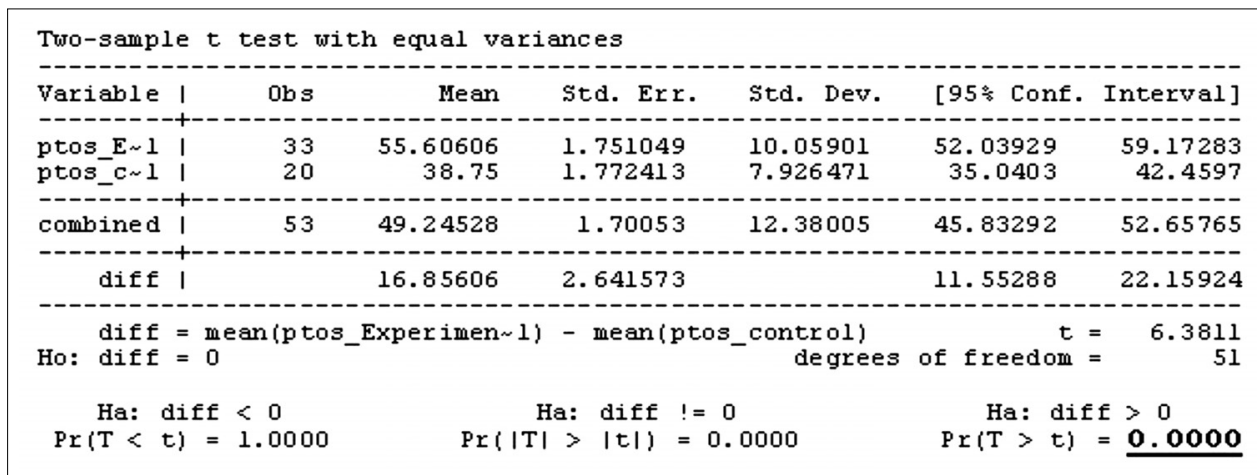

Dentro de este apartado también se analizará la diferencia existente entre la proporción de aprobados y suspensos por cada grupo. En relación con esto, en el grupo experimental hubo 31 aprobados $(93,9 \%)$ y 2 suspensos $(6,1 \%)$, mientras que en el grupo control hubo 13 aprobados (65\%) y 7 suspensos (35\%). Descriptivamente la proporción entre aprobados y suspensos es favorable al grupo experimental (existen proporcionalmente más aprobados que suspensos en dicho grupo), cuestión que se ratifica con la prueba de proporciones para dos muestras, pues, con un valor $z=-2,720$ y una significancia de $p=0,003$ (tabla 5), es posible decidir $y$, por lo tanto, afirmar que existe una diferencia significativa en las proporciones de ambas muestras. Gracias a lo anterior es posible sostener que la proporción de aprobados es significativamente mayor en el grupo experimental que en el grupo control.

Tabla 5. Prueba de proporciones para dos muestras

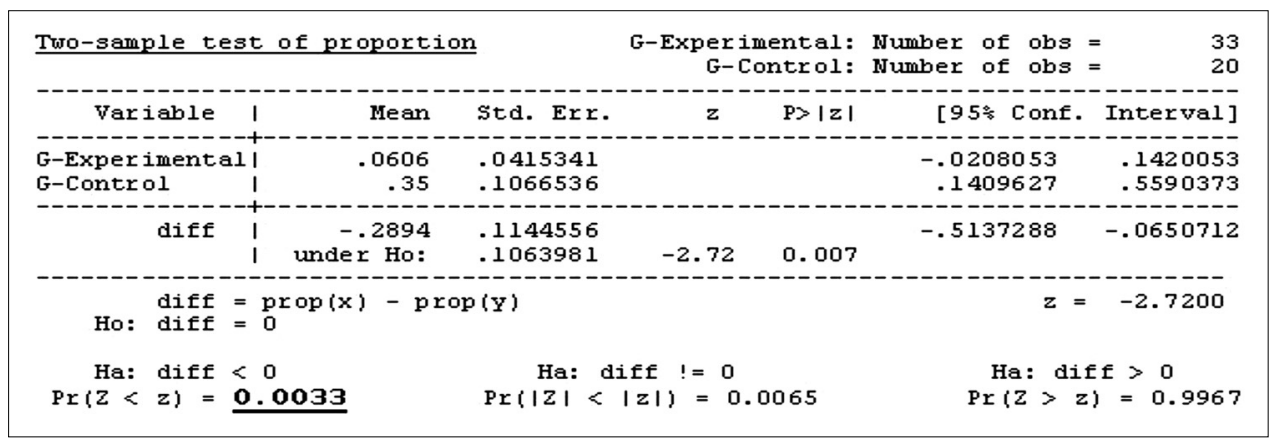

A la luz de todo lo expuesto previamente, es posible contrastar la hipótesis y sostener que efectivamente la incorporación de elementos extra textuales (ilustraciones, sonido, etc.) facilita la comprensión lectora de textos narrativos en L2. Lo anterior es una evidencia a favor de la idea de que la inclusión de elementos extra textuales (formato multimodal) en las pruebas de comprensión de textos narrativos en inglés se asocia a mejores rendimientos en los estudiantes a los cuales se las aplica. 


\section{DisCUSIÓN DE RESULTADOS}

Este estudio ha servido para confirmar la importancia de la variable multimodal en el aprendizaje de lenguas extranjeras, especialmente en la evaluación lectora de textos narrativos en L2, donde se ha ido integrando un modelo más interactivo de lectura, que está más relacionado con los textos multimodales, que dominan la escena fuera del aula, y que apoyan la construcción de significado (Kress, 2003). Dado los resultados comparativos entre ambos grupos, en este estudio se ha demostrado que los elementos extra textuales ayudarían a construir significado. Al respecto, Bernhardt (1983, 1991), concluye en sus investigaciones que los métodos tradicionales de evaluación lectora en L2 son insuficientes para capturar los mecanismos tan complejos que tienen lugar entre el aprendiz y el texto, encontrando, además, que el vocabulario no es el único predictor de la comprensión lectora en L2, ya que los textos multimodales (imágenes y video) son más efectivos que las narraciones planas.

En este contexto, los resultados del presente estudio parecen indicar que la hipótesis de investigación se confirma y aparecen razones para incluir textos en formato multimodal en la evaluación de la comprensión lectora en L2, por lo que consideramos que, a pesar de sus limitaciones metodológicas (distribución no aleatorizada de los sujetos y selección de textos narrativos comunicativos generales), este estudio puede contribuir a la toma de decisiones metodológicas y evaluativas, especialmente relacionadas con la integración de ambientes multimodales para la enseñanza y aprendizaje de L2, pues, como señala Serafini $(2011,2012)$, la incorporación de imágenes en los textos ayuda a que los lectores infieran significado fuera del texto.

La variable multimodal, entendida como la integración de imágenes, sonido, vOZ y movimiento facilitaría la captura de significados de textos narrativos en L2. Adicionalmente, es posible señalar que, dado los resultados de aprobados en el grupo experimental, los elementos extra textuales posibilitarían la interacción de los estudiantes con el texto, lo que se traduciría en mejores niveles de comprensión lectora, como así lo plantean estudios previos, tales como los hallazgos de Chun y Plass (1996), que sugieren que los elementos visuales y, particularmente, el video son facilitadores efectivos de la comprensión lectora en L2.

Lo anterior sugiere que los profesores de inglés, especialmente de los primeros niveles de aprendizaje de L2, deberían utilizar estrategias para hacer más significativo tanto el aprendizaje de L2 como su evaluación. Como se ha demostrado en este estudio, la variable multimodal permite establecer diferencias significativas entre los niveles de comprensión lectora alcanzado por los aprendices de L2; estas diferencias parecen indicar la necesidad de integrar la multimodalidad en los procesos de micro implementación curricular.

Adicionalmente, diversas investigaciones previas sugieren que la presentación multimodal de información constituye un facilitador de la comprensión lectora en L2 (Mayer y Sims, 1994; Chun y Plass, 1996). Así, los resultados del presente estudio indican que evaluar la comprensión lectora en L2 con modos verbales y visuales mejora las tasas de aprobados de pruebas que evalúan la referida destreza lingüística. 
Dada las limitaciones metodológicas del estudio, en futuras investigaciones se podrían implementar diseños en donde la variable ambiente multimodal pueda ser controlada y, de esa forma, no solamente poder extraer conclusiones sobre la relación entre textos multimodales y la comprensión lectora en L2, sino sobre la estrategia multimodal misma. Complementariamente, se podría indagar si los ambientes multimodales también facilitan el desarrollo de las destrezas auditiva y escrita, a través de ejercicios interactivos con apoyo multimodal. Adicionalmente, se podría intentar clarificar si existe relación entre la adquisición de vocabulario y la habilidad lectora, a través de textos narrativos con elementos multimodales. Finalmente, se podría investigar sobre la naturaleza de la interacción que realiza el lector entre el texto y los elementos extra textuales, y su relación con el procesamiento descendente (top-down) y ascendente (bottom-up) en el proceso lector en L2.

\section{IMPLICACIONES PEDAGÓGICAS}

De lo expresado anteriormente, parece pertinente abordar la necesidad de integrar elementos multimodales en textos orientados a desarrollar la comprensión lectora en L2, especialmente cuando se trata de estudiantes con un nivel umbral en L2. Este aspecto es especialmente importante cuando se desarrolla la comprensión lectora de textos narrativos en L2. En aprendices de L2, esto significa, además, que la comprensión lectora de textos narrativos ha de evaluarse en coherencia constructiva con las estrategias metodológicas utilizadas en el proceso lector, a partir de textos graduados (con intervención pedagógica), en formato multimodal, más que a través de textos auténticos y planos (sin intervención pedagógica), pues la imagen juega un papel importante en la interacción que tienen los estudiantes en la lectura comprensiva en L2, ya que a mayor integración de elementos extra textuales, parece facilitarse el nivel de comprensión lectora.

En este sentido, creemos que los textos en L2 necesitan ser intervenidos con recursos multimodales que faciliten su lectura comprensiva, buscando siempre las formas más apropiadas para que los estudiantes puedan procesar y construir significado, de manera mucho más activa y natural. Lo anterior requiere que la agenda de investigación en torno al tema de la multimodalidad convoque no sólo a los profesores de inglés, sino también a los investigadores vinculados con la lingüística aplicada, pues se necesita seguir explorando en el impacto que los ambientes multimodales y las herramientas de la multimedia interactiva tienen actualmente en la enseñanza y aprendizaje de L2, independientemente del enfoque que se aplique en el aula, sea éste EFL o ESP.

Desde esta perspectiva, creemos que los textos narrativos en L2, tanto auténticos como graduados, deben incluir elementos extra textuales multimodales, como elementos facilitadores de la interacción del estudiante con el texto. Este aspecto puede contribuir a que los profesores de inglés consideren la coherencia constructiva, que debe existir entre los textos de estudio utilizados durante el proceso de aprendizaje de L2, los enfoques metodológicos y las estrategias evaluativas, que se emplean en la micro implementación curricular, pues son claves para la comprensión y producción de L2. 
Por otra parte, creemos que para lograr los objetivos de una enseñanza de L2 efectiva y en consonancia con una generación de estudiantes mucho más multimodales, los profesores de inglés necesitan considerar de qué manera el uso de elementos extra textuales y la incorporación de elementos multimediales influyen en la interacción del lector con el texto. Esto demanda evaluar el aprendizaje multimodal y reformular el currículo de inglés de acuerdo a las nuevas innovaciones y a los sistemas de comunicación que están imperando fuera del aula.

\section{REFERENCIAS BIBLIOGRÁFICAS}

ALBERS, P. (2007). Visual discourse analysis: An introduction to the analysis of schoolgenerated visual texts. En D. W. Rowe, R. T. Jiménez, D. L. Compton, D. K. Dickinson, Y. Kim, K. M. Leander y V. J. Risko (Eds.), 56th yearbook of the National Reading Conference (pp. 81-95).

AUSUBEL, D.; NOVAK, J. y HANESIAN, H. (2005). Psicología educativa. Un punto de vista cognoscitivo. México, D.F.: Trillas.

BARNETT, M. A. (1989). More than meets the eye: Foreign language reading. NJ: Pretince Hall.

BERNHARDT, E. B. (1983). Testing foreign language reading comprehension. The immediate recall protocol. Die Unterrichichtspraxis, 16, 27-33.

BERNHARDT, E. B. (1991). Reading development in a second-language. Norwood, NJ: Ablex.

CARREL, P. L. y CARSON, J. G. (1997). Extensive and intensive reading in an EAP setting. English for Specific Purposes, 16, 47-60.

CHUN, D. y PLASS, J. (1996). Facilitating Reading comprehension with multimedia. System, 24 (4), pp. 503-519.

GEE, J. P. (2007). What video games have to teach us about learning and literacy. (Rev. ed.). Nueva York: Palgrave Macmillan.

HAMPEL, R. (2002). Network-based language teaching today: Multimodality, multidimensionality and interaction. Proceedings of the International Conference on Computers in Education (ICCE'02). The Open University, UK. Disponible en: http://csdl2.computer.org/comp/proceedings/icce/2002/1509/00/15090639.pdf.

HOSENFELD, C. (1979). A learning-teaching view of second language acquisition. Foreign Language Annals, 12, 51-54.

JEWITT, C. y KRESS, G. (2003). Multimodal literacy. Nueva York: Peter Lang.

KODA, K. (1994). Second language reading research: problems and possibilities. Applied Psycholinguistics, 15, 1-28.

KRESS. G. (2003). Literacy in the New Media Age. Cambridge: The Cromwell Press. KRESS, G. y VAN LEEUWEN, T. (2001.) Multimodal Discourse. Londres: Arnold.

LAUFER, B. (1986). Possible changes in attitude towards vocabulary acquisition research. International Review of Applied Linguistics, 24 (1), 69-75.

MATTHEWMAN, S.; BLIGHT, A. y DAVIES, C. (2004). What does multimodality mean for English? Creative tensions in teaching new texts and new literacies. 
Education, Communication and Information, 4 (1). Disponible en: http:// www.interactiveeducation.ac.uk/out_mat.pdf.

MAYER, R. y SIMS, V. K. (1994). For whom is a picture worth a thousand words? Extensions of dual coding theory of multimedia learning. Journal of Educational Psychology, 86 (3), 389-401.

PALINCSAR, A. S. y BROWN, A. L. (1984). Reciprocal teaching of comprehensionfostering and comprehension-monitoring activities. Cognition and Instruction, 1, 117-175.

RODRÍGUEZ, W.; OCHOA, S. y PARKER, R. (2006). The crosslinguistic role of cognitive academic language proficiency on reading growth in Spanish and English. Bilingual Research Journal, 30. Disponible en: http://brj.asu.edu/vol30_no1/art5.pdf.

SCHOONEN, R.; HULSTIJN, J. y BOSSERS, B. (1998). Metacognitive and language specific knowledge in native and foreign language reading comprehension: An empirical study among Dutch students in grades 6, 8 and 10. Language Learning, 48, 71-106.

SERAFINI, F. (2011). Expanding perspectives for comprehending visual images in multimodal texts. Journal of Adolescent and Adult Literacy, 54, 342-350.

SERAFINI, F. (2012). Expanding the four resources model: Reading visual and multimodal texts. Pedagogies: An International Journal, 7 (2), 150-164.

SUSSER, B. y ROBB, TH. (1990). EFL extensive reading instruction: Research and procedure. JALT Journal, 12 (2). Disponible en: http://www.cc.kyoto-su.ac.jp/ $\sim$ trobb/sussrobb.html.

WIDDOWSON, H. G. (1978). Teaching language as communication. Oxford: Oxford University Press.

WILLOWS, D. M. y HOUGHTON, H. A. (1987). The Psychology of illustration. Nueva York: Springer. 